\title{
Editorial \\ Androgens in rheumatoid arthritis: when are they effectors?
} Maurizio Cutolo

Research Laboratory and Academic Unit of Clinical Rheumatology, Department of Internal Medicine, University of Genova Italy, Viale Benedetto XV, 6, 16132 Genova Italy

Corresponding author: Maurizio Cutolo, mcutolo@unige.it

Published: 22 September 2009

Arthritis Research \& Therapy 2009, 11:126 (doi:10.1186/ar2804)

This article is online at http://arthritis-research.com/content/11/5/126

(c) 2009 BioMed Central Ltd

See related research by Karlson et al., http://arthritis-research.com/content/11/3/R97

\begin{abstract}
Neither hormone receptor genes nor plasma androgens seem significantly altered in female subjects before they became affected by rheumatoid arthritis (RA) and, therefore, do not seem to play a role as risk factors for its development. However, serum testosterone levels are inversely correlated with RA activity and dehydro-epiandrosterone sulfate (DHEAS) plasma levels are inversely correlated with both disease duration and clinical severity in patients already affected by active RA. In particular, gonadal and adrenal androgens (that is, testosterone and DHEAS) are significantly decreased in inflamed synovial tissue/fluids during active disease as a consequence of the inflammatory reaction, which supports a pro-inflammatory milieu in RA joints. Recently, male gender has been found to be a major predictor of remission in early RA.
\end{abstract}

\section{Introduction}

The study by Karlson and colleagues in Arthritis Research and Therapy [1] confirms that neither hormone receptor genes nor plasma androgens play a role as risk factors for the development of rheumatoid arthritis (RA), at least in female subjects. On the contrary, clinical and experimental evidence seems to support perturbations in peripheral androgen metabolism and a modulatory role for estrogens in patients with active and overt RA [2].

\section{Androgens in active rheumatoid arthritis}

Clinical and epidemiological evidence supports that androgens protect more male than female subjects from the development of immune-inflammatory diseases [3]. Androgens exert anti-inflammatory activities, at least at the level of the RA synovial tissue, which contrast with the immune-enhancer activities locally exerted by estrogens and their metabolites [3]. It is well known that serum testosterone levels are inversely correlated with RA disease activity and dehydroepiandrosterone sulfate (DHEAS) levels are inversely correlated with both disease duration and clinical severity [4].

Recently, male gender has been found to be a major predictor of remission in early RA [5]. Although disease activity was not obviously more pronounced in female RA patients at the onset of disease, the disease course became markedly worse in women. Disparity in RA remission frequencies between women and men could not even be explained by differences in disease duration, age or treatment with disease-modifying antirheumatic drugs or glucocorticoids, but the probability of achieving a treatment response, at least with methotrexate or anti-TNF drugs, is reduced by 35 to $50 \%$ in women [5]. Again, just as in active RA, the presence of androgens (equivalent to being a male patient) seems to indicate better prognosis.

It seems, therefore, that the effects of TNF antagonists (and, generally, anticytokine agents) on the levels of peripheral sex hormones are exerted more quickly in RA synovial tissue than in serum. In synovial tissue, TNF antagonists seem to downregulate the increased conversion of androgens (anti-inflammatory) to estrogens (immune response enhancers) that is induced by the aromatase complex [6]. As is known, inflammatory cytokines, such as TNF, are inducers of the aromatase complex [4].

The beneficial effects of restoring levels of synovial tissue androgens might be clinically more evident in male RA patients because they suffer more intensively from the inflammation-related decrease of androgens, owing to the stimulatory action of TNF on the conversion of androgens into estrogens in synovial tissue [6]. Indeed, men with active RA have lower synovial fluid testosterone levels and higher levels of estradiol compared to healthy subjects as a result of increased synovial tissue production of estrone [7].

\section{Androgen changes in RA: chicken or egg?}

The crucial question is: does inflammation lead to reduction of androgen levels (through conversion) or does the sex hormonal environment influence inflammation? The answer is that inflammation clearly downregulates androgen production, but estrogens, and in particular selected hydroxylated

$\mathrm{DHEAS}=$ dehydro-epiandrosterone sulfate; $\mathrm{RA}=$ rheumatoid arthritis; TNF = tumour necrosis factor. 


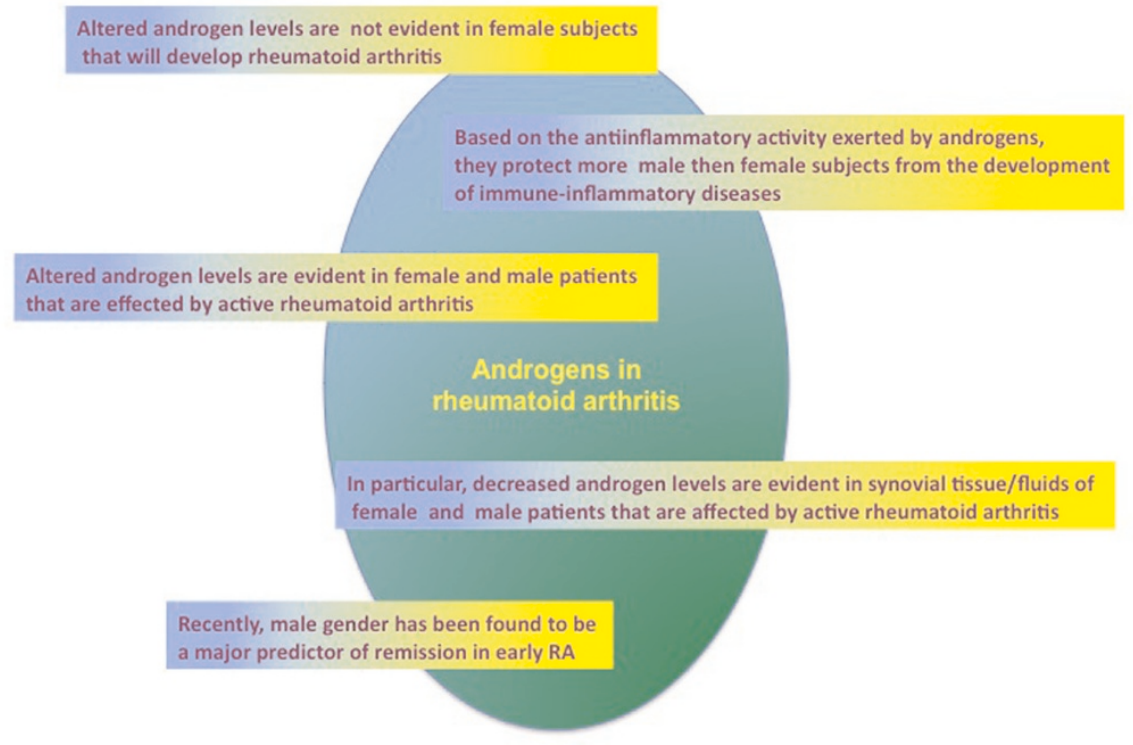

Principal facts characterizing the roles of androgens in rheumatoid arthritis patients.

estrogen metabolites, enhance the immune-inflammatory response, at least in RA [8].

Interestingly, treatment with anti-estrogens (that is, toremifene and tamoxifen) inhibited the differentiation of cultured RA synovial macrophages into dendritic cells and the capacity of synovial-macrophage-derived dendritic cells to stimulate allogeneic $T$ cells [9]. In contrast, a small randomizedcontrolled trial of testosterone treatment demonstrated significantly improved symptoms in men with RA [10].

Therefore, the research questions that Karlson and colleagues' results now pose are, in reality, only a further confirmation that the real higher risk for developing RA and autoimmune rheumatic diseases in general, based on sex hormone levels, is to be female because of the related estrogenic hormonal patterns $[1,2]$.

\section{Adrenal and gonadal androgen relationships}

Activation of the hypothalamus-pituitary-adrenal axis by proinflammatory stimuli and chronic stress leads to a parallel decrease in hypothalamus-pituitary-gonadal axis activity $[2,11]$. This can be substantiated by decreased levels of follicle-stimulating hormone and luteinizing hormone, and it is even more evident by looking at the levels of serum testosterone and the serum adrenal androgen DHEAS [2]. During a chronic inflammatory process like active RA, levels of both serum testosterone and, in particular, serum DHEAS become lower. Since testosterone and its precursors DHEAS and DHEA have anti-inflammatory properties, the decline in levels of these hormones further supports the proinflammatory process.
In the adrenal and gonadal glands, the loss of DHEA and DHEAS is attributed to a synthetic blockade of the second step of the enzyme P450c17, again induced by inflammatory cytokines such as IL1 $\beta$ and TNF. Increased DHEAS levels during treatment with TNF antagonists in active RA patients suggest an improved adrenal function [12].

\section{Conclusions}

Neither plasma androgens nor hormone receptor genes seem significantly altered in female subjects that will became affected by RA; therefore, they do not seem to play a role as risk factors for the development of RA. However, adrenal and gonadal androgens, which exert anti-inflammatory activities, are significantly decreased in inflamed tissues (that is, synovial fluid) during active RA in both male and female patients, which supports a pro-inflammatory milieu at least in RA joints (Figure 1). Interestingly, increased aromatization of androgens has been demonstrated in cultured synovial cells from RA patients and the synthesized estrogens are further converted to pro-proliferative estrogens, such as the 16hydroxylated forms of estrone and $17 \beta$-estradiol [8].

\section{Competing interests}

The author declares that they have no competing interests.

\section{References}

1. Karlson EW, Chibnik LB, McGrath M, Chang SC, Keenan BT, Costenbader KH, Fraser PA, Tworoger S, Hankinson SE, Lee IM, Buring J, De Vivo I: A prospective study of androgen levels, hormone related genes and risk of rheumatoid arthritis. Arthritis Res Ther 2009, 11:R97.

2. Cutolo M, Straub RH: Insights into endocrine-immunological disturbances in autoimmunity and their impact on treatment. Arthritis Res Ther 2009, 11:218. 
3. Cutolo M, Straub RH, Bijlsma JW: Neuroendocrine-immune interactions in synovitis. Nat Clin Pract Rheumatol 2007, 3:627634.

4. Gordon D, Beastall GH, Thomson JA, Sturrock RD: Prolonged hypogonadism in male patients with rheumatoid arthritis during flares in disease activity. $\mathrm{Br} J$ Rheumatol 1988, 27:440444.

5. Forslind K, Hafström I, Ahlmén M, Svensson B; BARFOT Study Group: Sex: a major predictor of remission in early rheumatoid arthritis? Ann Rheum Dis 2007, 66:46-52.

6. Straub $\mathrm{RH}$, Härle $\mathrm{P}$, Sarzi-Puttini $\mathrm{P}$, Cutolo $\mathrm{M}$ : Tumor necrosis factor-neutralizing therapies improve altered hormone axes: an alternative mode of antiinflammatory action. Arthritis Rheum 2006, 54:2039-2046.

7. Cutolo M, Sulli A, Capellino S, Villaggio B, Montagna P, Pizzorni C, Paolino S, Seriolo B, Felli L, Straub RH: Anti-TNF and sex hormones. Ann N Y Acad Sci 2006, 1069:391-400.

8. Capellino S, Montagna P, Villaggio B, Soldano S, Straub RH, Cutolo $\mathrm{M}$ : Hydroxylated estrogen metabolites influence the proliferation of cultured human monocytes: possible role in synovial tissue hyperplasia. Clin Exp Rheumatol 2008, 26:903909.

9. Komi J, Möttönen M, Luukkainen R, Lassila O: Non-steroidal antioestrogens inhibit the differentiation of synovial macrophages into dendritic cells. Rheumatology (Oxford) 2001, 40: 185-191.

10. Cutolo M, Balleari E, Giusti M, Intra E, Accardo S: Androgen replacement therapy in male patients with rheumatoid arthritis. Arthritis Rheum 1991, 34:1-5.

11. Cutolo M, Straub RH: Stress as a risk factor in the pathogenesis of rheumatoid arthritis. Neuroimmunomodulation 2006, 13: 277-282.

12. Ernestam S, Hafström I, Werner S, Carlström K, Tengstrand B: Increased DHEAS levels in patients with rheumatoid arthritis after treatment with tumor necrosis factor antagonists: evidence for improved adrenal function. J Rheumatol 2007, 34: 1451-1458. 